\title{
Characteristics of dispersed organic matter of the Menilite Beds from the Dukla Unit based on microscopic analysis and Rock-Eval pyrolysis
}

\author{
Charakterystyka rozproszonej materii organicznej warstw menilitowych jednostki \\ dukielskiej na podstawie analiz mikroskopowych i pirolizy Rock-Eval
}

\author{
Konrad Ziemianin \\ Oil and Gas Institute - National Research Institute
}

\begin{abstract}
Organic matter from Menilite Beds of the Dukla Unit was studied (optical microscopy, Rock-Eval pyrolysis). In its composition, macerals from vitrinite, liptinite and inertinite groups are observed. Macerals of the liptinite group are dominating (alginite, bituminite and liptodetrinite). Among all invested lithological types, mudstones have the highest average content of these macerals, while in case of marly shales, sandstones/siltstones, micrite limestones and chert the content of individual liptinite macerals is always lower than $1 \mathrm{vol} \%$. The vitrinite group is represented mainly by collotelinite and vitrodetrinite and its average content in all analyzed lithologies does not exceed $1 \mathrm{vol} \%$. Fragments of collotelinite show significant diversity in terms of the reflectance. Macerals of the inertinite group (fusinite, semifusinite, inertodetrinite) are usually limited to single occurrences within the entire investigated sample area. In 41 out of 43 samples, the collotelinite fragments were numerous enough that it was possible to measure the reflectance. For samples in which reflectance of vitrinite fragments was measured, this parameter ranges from $0.25 \%$ to $0.56 \%$. Rock-Eval pyrolysis results provided additional information, of which the most important were values of TOC (from $0.47 \%$ to $6.04 \%$, average $2.61 \%$ ), $\mathrm{T}_{\max }\left(417-449^{\circ} \mathrm{C}\right)$, hydrogen $(95-502)$ and oxygen $(<133)$ indices. In the western part of the investigated area, within the tectonic window of Światkowa, Menilite Beds are in the phase of oil window, while in other locations organic matter is immature. Analyzed samples contain types II, II/III or III of kerogen. Type II dominates in clay shales and mudstones, while type III is most often observed in marly and carbonate shales and also in sandstones/siltstones.
\end{abstract}

Key words: menilite shales, alginite, bituminite, vitrinite reflectance, Rock-Eval pyrolysis.

STRESZCZENIE: Przebadano fragmenty rozproszonej materii organicznej z warstw menilitowych jednostki dukielskiej (mikroskopia optyczna, piroliza Rock-Eval). W jej składzie obserwuje się macerały z grupy witrynitu, liptynitu i inertynitu, przy czym macerały z grupy liptynitu (alginit, bituminit i liptodetrynit) są dominujące. Najwyższy średni udział macerałów z grupy liptynitu w obrębie wszystkich przebadanych odmian litologicznych obserwuje się w mułowcach, podczas gdy w łupkach marglistych, piaskowcach, pyłowcach, wapieniach mikrytowych czy rogowcach zarówno alginit, jak i bituminit i liptodetrynit nie przekraczają $1 \%$ obj. Grupa macerałów witrynitu jest reprezentowana przez kolotelinit i witrodetrynit, jednak ich średni udział nigdy nie przekracza $1 \%$ obj. wszystkich składników w skale. Fragmenty kolotelinitu są zróżnicowane pod kątem refleksyjności. Macerały grupy inertynitu (fuzynit, semifuzynit, inertodetrynit) są zwykle ograniczone jedynie do pojedynczych wystąpień. W przypadku 41 z 43 próbek fragmenty kolotelinitu były na tyle liczne, że możliwy był pomiar ich refleksyjności, która mieściła się w przedziale od $0,25 \%$ do 0,56\%. Wyniki analizy mikroskopowej uzupełniono o dane z pirolizy Rock-Eval. Udział materii organicznej (parametr TOC) waha się od $0,47 \%$ do $6,04 \%$ (przy średniej na poziomie 2,61\%). Parametr Tmax zawiera się w przedziale od $417^{\circ} \mathrm{C}$ do $449^{\circ} \mathrm{C}$, indeks wodorowy HI w przedziale od 95 do 502, natomiast indeks tlenowy OI nie przekracza 133. We wschodniej części przebadanego obszaru (okno tektoniczne Świątkowej) materia organiczna wkroczyła w fazę przemian termicznych odpowiadającą tzw. oknu ropnemu, natomiast na pozostałym obszarze jest niedojrzała. Przebadane próbki zawierają kerogen typu II, III, jak również ich mieszaninę. Typ II dominuje w łupkach ilastych i mułowcach, podczas gdy typ III jest najczęściej obserwowany w łupkach marglistych i węglanowych, jak również w piaskowcach i pyłowcach.

Słowa kluczowe: łupki menilitowe, alginit, bituminit, refleksyjność witrynitu, piroliza Rock-Eval.

Corresponding author: K. Ziemianin, e-mail: konrad.ziemianin@inig.pl

Article contributed to the Editor 21.01.2019. Approved for publication 25.04.2019 r. 


\section{Introduction}

The Menilite Beds were first described by Glocker in Moravia in 1843 (Świdziński, 1947). Since then, they have become the subject of intensive research. Their high organic matter content and wide distribution within the Carpathian area are the reasons why they play a key role in analyzing petroleum systems in this region. At the beginning of the 1990s, the rate and conditions of deposition based on Rock-Eval pyrolysis as well as analysis of sulfur content and carbon-sulfur ratio were determined by Vetö and Hetényi (1991), while the maturation and type of kerogen for menilite shales from Ukraine were characterized by Koltun (1992). In the similar period, the geochemical studies of the Menilite Beds were carried out by ten Haven et al. (1993), pointing to genetic relationships between analyzed oil and potential source rocks. In turn, Matyasik (1994) performed geochemical characterization of menilite samples from the Skole Unit. The increase in interest in the menilite shales generation potential results in subsequent publications on the subject (Köster at al., 1995, 1998a; Kruge et al., 1996; Matyasik and Kupisz, 1996; Koltun et al., 1998). Analysis of biomarkers and carbon isotopes become the basis for attempts to reconstruct the palaeoenvironment (Köster at al., 1996, 1998b). Although generally very good (Köster at al., 1998a) or even excellent menilite shales generation potential (Kruge et al., 1996), in 2001 Matyasik et al. indicated that the degree of maturity of menilite shales is insufficient to consider them as the only source of oil in the Carpathians. The origin of the oil originating from the menilite shales, as well as the relationship between organic sulfur and the kinetics of the hydrocarbon generation processes was described by Więcław in 2002.
The kinetics of the oil generation process from type II and IIS kerogen from menilite shales were also investigated by Lewan et al. (2006). In a number of publications (Kotarba and Lewan, 2002, Curtis et al., 2004, Kotarba et al., 2005), the subject of correlation of petroleum and source rocks was investigated based on water pyrolysis of the menilite shales. The problem of the mentioned correlations and the generation potential of menilite shales of both Polish and Ukrainian part of the Carpathians was developed by Kotarba et al in 2007 (authors studied rock, oil and gas samples). The issue of the composition and genesis of gas from the Menilite Beds was discussed in detail by Kotarba and Nagao (2008) and Kotarba et al. (2009). Menilite shales were described as the main source of hydrocarbons in the Carpathians by Pawlewicz (2006) and Schasenhofer and Koltun (2012). The issue of the source and occurrence of hydrocarbons in the Carpathian region was also investigated by Kotarba and Koltun (2006). For selected profiles in the eastern part of the Outer Carpathians, attention was paid to the vertical variation in the maturity indexes (Więcław et al., 2008). The results of the menilite shales maturity investigations were summarized in 2009 by Kosakowski et al. The authors investigated large number of samples of the menilite rocks $(>550)$ and confirmed that Menilite Beds are the best source rocks and the type II kerogen present in them is generally immature or mature in the early stage of the “oil window”. The newer publications of Kotarba et al. (2013, 2014) also confirmed the best generation potential of menilite shales. Recently, the influence of changes of deposition facies on the generation potential of the Menilite Beds was examined (Kosakowski et al., 2018) and it was found that there is no direct correlation in this case.

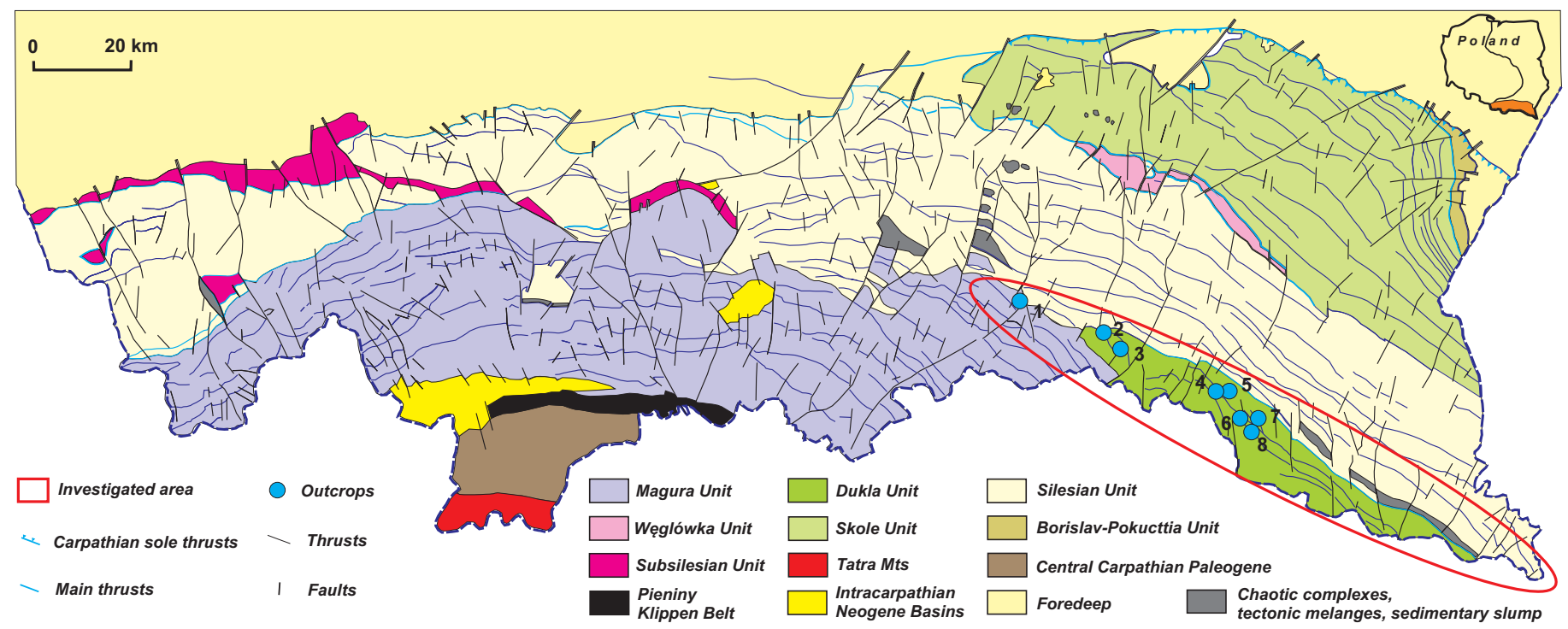

Fig. 1. Area of investigation with the location of the outcrops (Jankowski 2008, Jankowski, Probulski, 2011, modified). Outcrops: 1 Świątkowa Wielka, 2 - Mszana, 3 - Zyndranowa, 4 - Wisłok Wielki, 5 - Izwór Stream, 6 - Czystogarb, 7 - Komańcza-1, 8 - Komańcza-2

Rys. 1. Obszar badań (Jankowski 2008, Jankowski, Probulski, 2011, zmieniony). Odsłonięcia: 1 - Świątkowa Wielka, 2 - Mszana, 3 - Zyndranowa, 4 - Wisłok Wielki, 5 - potok Izwór, 6 - Czystogarb, 7 - Komańcza-1, 8 - Komańcza-2 
Geochemical interpretations are not complete unless they are supplemented with optical characteristics of dispersed organic matter. Unfortunately, petrography of organic matter in the case of the Menilite Beds is often either completely marginalized or limited only to vitrinite reflectance measurements. Therefore, publications where the results of microscopic investigations of organic matter are presented are rather rare (Koltun, 1992; Kruge et al., 1996; Kotulva, 2004; Semyrka, 2009; Kosakowski et al., 2009, 2018; Zielińska, 2012; Kotarba et al., 2013, Waliczek et al., 2017, Wendorff et al., 2017).

This paper is focused on microscopic characterization of organic matter, while the results of geochemical analyzes (RocEval pyrolysis) are used here only to make it more complete. The aim of this paper is to present the maceral composition of Menilite Beds within the Dukla Unit, while organic matter composition of Menilite Beds within Skole and Silesian units were a subject of previous papers (Ziemianin, 2017, 2018).

\section{Field work and research material}

The research covered the area of the Polish part of the Dukla Unit (Fig. 1). Samples (43) were collected from 8 outrocps of the Menilite Beds (Świątkowa Wielka, Mszana, Zyndranowa, Wisłok Wielki, Izwór stream, Czystogarb, and 2 outrocrops from the Komańcza region).

The collected rocks represented a variety of the lithological types (Fig. 2) - mainly various shale types (clay, marly or carbonate) and in smaller quantities mudstones, siltstones, sandstones and sporadically cherts and micrite limestones.

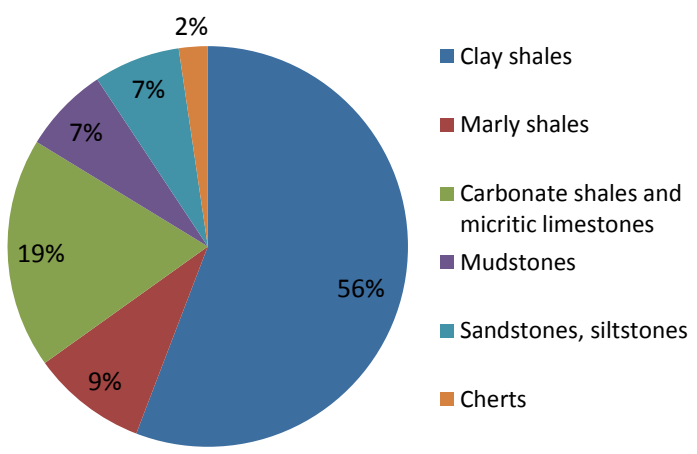

Fig. 2. Content of different lithological types within the collected samples

Rys. 2. Udział różnych typów litologicznych wśród zebranych próbek

\section{Methods}

For microscopic analysis polished samples and thin plates were prepared. Observations were made in reflected light and fluorescence mode (analysis of maceral composition and vitrin- ite reflectance measurements), as well as in transmitted light (determination of lithological type and mineral composition). Observations in reflected light and fluorescence mode were performed with the use of Carl Zeiss Axioplan microscope, at magnification 500x, in immersion (oil immersol $518 \mathrm{~N}$, $\mathrm{n}=1.518$ ), while observations in transmitted light were made using a Nikon Eclipse LV100 POL microscope, at magnifications in range from $50 \mathrm{x}$ to $1000 \mathrm{x}$. In order to determine the maceral composition, a planimetric analysis was performed (at least 500 points were counted). Measurements of vitrinite reflectance were also performed. During the measurements redeposited grains (with higher reflectance) as well as those saturated with lipid substances (lowering reflectance) were avoided. Before the measurements were made, the microscope was calibrated using three standards of defined reflectance (spinel $\mathrm{R}_{\mathrm{o}}=0.421 \%$, sapphire $\mathrm{R}_{\mathrm{o}}=0.593 \%$, garnet $\left.\mathrm{R}_{\mathrm{o}}=0.900 \%\right)$. The results were supplemented with photographic documentation.

Additionally, Rock-Eval pyrolytic analysis was carried out using Rock-Eval-6 model (standard equipment). A number of parameters have been obtained, among which most important were: TOC (total organic carbon content), $\mathrm{T}_{\max }$ (temperature at the point of maximum hydrocarbon generation), hydrogen index HI (calculated as S2/TOC, expressed in $\mathrm{mg}$ of hydrocarbons per g TOC), oxygen index OI (calculated as S3/TOC, expressed in $\mathrm{mg} \mathrm{CO}_{2}$ per $\mathrm{g}$ TOC).

\section{Results}

Organic matter most often occurs in the form of elongated laminae or fine, dispersed fragments with a slight elongation in the studied rocks. The content of organic matter (determined on the basis of Rock-Eval pyrolysis results - TOC parameter) ranges from $0.47 \%$ to $6.04 \%$ (average $2.61 \%$ ). In its composition, macerals from vitrinite, liptinite and inertinite groups are observed (Tab. 1).

Macerals of the liptinite group are dominating. Within the macerals of this group, alginite, bituminite and liptodetrinite are observed. Alginite is most often observed in the form of a fine, elongated lamalginite (plate I, A) and slightly less frequently in the form of larger bodies - telalginite. Within the telalginite Tasmanites are observed (plate I, B). Both forms of alginite show yellow fluorescence (telalginite fluorescence appears to be more intense). The content of alginite ranges from individual occurrences up to $20 \mathrm{vol} \%$ of all components in the rock, with an average of about $2 \mathrm{vol} \%$. Bituminite forms more or less regular laminae (plate I, C). It exhibits brown fluorescence. Its maximum content reaches 5 vol\%., although there are samples in which it is not present at all. Liptodetrinite is composed of fine $(<10 \mu \mathrm{m})$ fragments 
Table 1. Maceral content in the investigated samples

Tabela 1. Udział poszczególnych macerałów w przebadanych próbkach

\begin{tabular}{|c|c|c|c|c|c|c|c|c|c|c|}
\hline \multirow{2}{*}{ Outcrop } & \multirow{2}{*}{ Lithology } & \multirow{2}{*}{$\begin{array}{c}\text { Sample } \\
\text { ID }\end{array}$} & \multicolumn{2}{|c|}{ Vitrinite } & \multicolumn{3}{|c|}{ Inertinite } & \multicolumn{3}{|c|}{ Liptinite } \\
\hline & & & collotelinite & vitrodetrinite & fusinite & semifusinite & inertodetrinite & alginite & bituminite & liptodetrinite \\
\hline \multirow{4}{*}{ 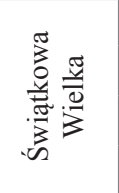 } & clay shale & 1 & TRACE & TRACE & n.o. & n.o. & trace & 0.16 & n.o. & 0.63 \\
\hline & clay shale & 2 & 0.46 & 0.46 & trace & trace & trace & 1.08 & n.o. & 0.46 \\
\hline & clay shale & 3 & 0.74 & 0.55 & trace & n.o. & trace & 0.18 & n.o. & 0.92 \\
\hline & marly shale & 4 & TRACE & 0.20 & n.o. & n.o. & trace & trace & n.o. & trace \\
\hline \multirow{5}{*}{$\begin{array}{l}\text { त्ञ } \\
\text { 党 }\end{array}$} & carbonate shale & 1 & trace & TRACE & trace & trace & trace & 0.79 & TRACE & 1.89 \\
\hline & sandstone & 2 & 1.28 & 0.32 & trace & trace & n.o. & 0.80 & 1.91 & TRACE \\
\hline & micritic limestone & 3 & TRACE & TRACE & n.o. & trace & trace & 0.33 & 0.33 & TRACE \\
\hline & carbonate shale & 4 & 0.79 & 0.16 & TRACE & trace & TRACE & 2.05 & 1.74 & 0.95 \\
\hline & micritic limestone & 5 & TRACE & 0.16 & n.o. & trace & n.o. & TRACE & n.o. & TRACE \\
\hline \multirow{6}{*}{ 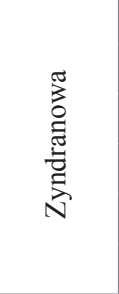 } & clay shale & 1 & 0.76 & TRACE & n.o. & n.o. & n.o. & 1.89 & 0.38 & 1.32 \\
\hline & clay shale & 2 & TRACE & 0.20 & n.o. & n.o. & n.o. & 2.57 & n.o. & 1.38 \\
\hline & clay shale & 3 & 0.18 & TRACE & n.o. & n.o. & n.o. & 0.55 & n.o. & 0.55 \\
\hline & clay shale & 4 & TRACE & TRACE & n.o. & n.o. & trace & 0.97 & 0.39 & 2.13 \\
\hline & clay shale & 5 & TRACE & TRACE & n.o. & n.o. & n.o. & 1.29 & n.o. & 0.74 \\
\hline & marly shale & 6 & 0.20 & 0.40 & n.o. & n.o. & n.o. & 1.60 & trace & 1,00 \\
\hline \multirow{6}{*}{ 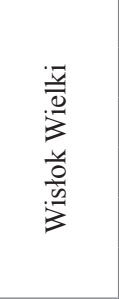 } & clay shale & 1 & 0.19 & 0.19 & trace & n.o. & trace & 1.69 & 1.69 & 0.94 \\
\hline & sandstone & 2 & trace & TRACE & n.o. & n.o. & n.o. & trace & n.o. & trace \\
\hline & clay shale & 3 & 0.39 & 0.78 & trace & n.o. & trace & 2.15 & 2.54 & 0.39 \\
\hline & carbonate shale & 4 & 0.20 & 0.40 & trace & n.o. & trace & TRACE & n.o. & 0.40 \\
\hline & clay shale & 5 & 0.38 & TRACE & trace & trace & trace & 1.34 & 1.15 & 0.95 \\
\hline & clay shale & 6 & TRACE & TRACE & trace & n.o. & n.o. & 1.90 & 5.14 & 1.52 \\
\hline \multirow{5}{*}{ 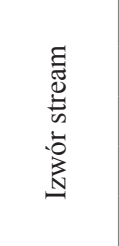 } & clay shale & 1 & TRACE & 0.19 & trace & n.o. & trace & 1.30 & 0.93 & 1.86 \\
\hline & clay shale & 2 & TRACE & 0.39 & trace & trace & trace & 1.96 & 0.39 & 0.98 \\
\hline & marly shale & 3 & TRACE & TRACE & n.o. & n.o. & n.o. & 0.38 & trace & TRACE \\
\hline & siltstone & 4 & 0.40 & TRACE & trace & n.o. & n.o. & 0.20 & 0.20 & TRACE \\
\hline & clay shale & 5 & 0.39 & TRACE & trace & n.o. & 0.2 & 0.98 & 2.16 & 0.39 \\
\hline \multirow{6}{*}{ 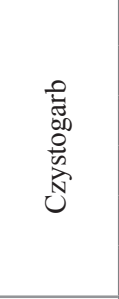 } & clay shale & 1 & TRACE & 0.39 & n.o. & n.o. & trace & 4.43 & 1.93 & 1.73 \\
\hline & clay shale & 2 & 0.20 & TRACE & n.o. & n.o. & n.o. & 7.23 & 3.41 & 0.40 \\
\hline & clay shale & 3 & 0.39 & TRACE & n.o. & n.o. & n.o. & 1.17 & 1.57 & 0.20 \\
\hline & chert & 4 & trace & TRACE & n.o. & n.o. & n.o. & TRACE & n.o. & 0.20 \\
\hline & mudstone & 5 & 0.37 & TRACE & trace & n.o. & n.o. & 2.61 & 5.03 & 0.56 \\
\hline & clay shale & 6 & TRACE & TRACE & trace & n.o. & n.o. & 0.60 & 3.79 & 0.60 \\
\hline \multirow{5}{*}{ 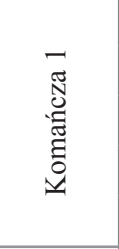 } & clay shale & 1 & 0.15 & TRACE & trace & trace & n.o. & 2.11 & 2.11 & 2.11 \\
\hline & clay shale & 2 & 0.17 & TRACE & trace & n.o. & n.o. & 2.19 & 4.22 & 1.18 \\
\hline & clay shale & 3 & 0.33 & TRACE & n.o. & n.o. & n.o. & 2.94 & 2.45 & 2.45 \\
\hline & clay shale & 4 & 0.16 & TRACE & trace & trace & trace & 0.47 & 4.1 & TRACE \\
\hline & mudstone & 5 & TRACE & TRACE & trace & n.o. & n.o. & 3.31 & 4.57 & 0.94 \\
\hline \multirow{6}{*}{ 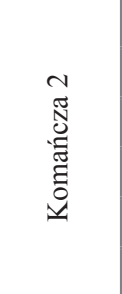 } & clay shale & 1 & TRACE & TRACE & n.o. & n.o. & n.o. & 13.16 & 0.85 & 2.22 \\
\hline & carbonate shale & 2 & 0.98 & 0.16 & n.o. & trace & n.o. & 1.14 & 0.33 & 0.33 \\
\hline & carbonate shale & 3 & 0.16 & 0.32 & trace & trace & n.o. & 0.49 & n.o. & 0.49 \\
\hline & carbonate shale & 4 & 0.16 & TRACE & trace & trace & trace & 1.28 & n.o. & 0.16 \\
\hline & mudstone & 5 & 0.16 & TRACE & trace & trace & n.o. & 20.39 & 0.49 & 2.63 \\
\hline & marly shale & 6 & 0.63 & 0.47 & trace & n.o. & n.o. & 1.26 & n.o. & 0.78 \\
\hline
\end{tabular}

TRACE - fragments often observed; trace - very few fragments; n.o. - not observed 


\section{PLATE I / TABLICA I}

A. Komańcza-2, sample 2. Lamalginite, exhibiting yellow fluorescence (arrow). Fluorescence mode A. Komańcza 2, próbka 2. Lamalginit wykazujący fluorescencję w kolorze żółtym (strzałka). Tryb fluorescencji

B. Komańcza-2, sample 5. Thickwalled telalginite - Tasmanites sp. (arrow). Fluorescence mode

B. Komańcza 2, próbka 5. Telalginit o grubych ścianach komórkowych Tasmanites sp. (strzałka). Tryb fluorescencji

C. Komańcza-1, sample 1. Bituminite laminae exhibiting brown fluorescence (arrows). Fluorescence mode

C. Komańcza 1, próbka 1. Laminy bituminitu o brązowej fluorescencji (strzałki). Tryb fluorescencji

D. Komańcza-1, sample 3. Association of lamalginite (green arrows) with fine liptodetrinite particle (red arrows), most probably of algae origin. Fluorescence mode

D. Komańcza 1, próbka 3. Asocjacja lamalginitu (zielone strzałki) z drobnymi fragmentami liptodetrynitu (strzałki czerwone) o najpewniej algowej genezie. Tryb fluorescencji

E. Zyndranowa, sample 6. Two vitrinite fragments with a different reflectance (arrows). Reflected light

E. Zyndranowa, próbka 6. Dwa fragmenty witrynitu o odmiennej refleksyjności (strzałki). Światło odbite

F. Komańcza-2, sample 6. Elongated fragment of saprovitrinite (arrow). Reflected light

F. Komańcza 2, próbka 6. Wydłużony fragment saprowitrynitu (strzałka). Światło odbite

G. Komańcza-2, sample 2. Fragment of fusinite with preserved cell structure (arrow). Reflected light

G. Komańcza 2, próbka 2. Fragment fuzynitu z zachowaną strukturą tkankową (strzałka). Światło odbite
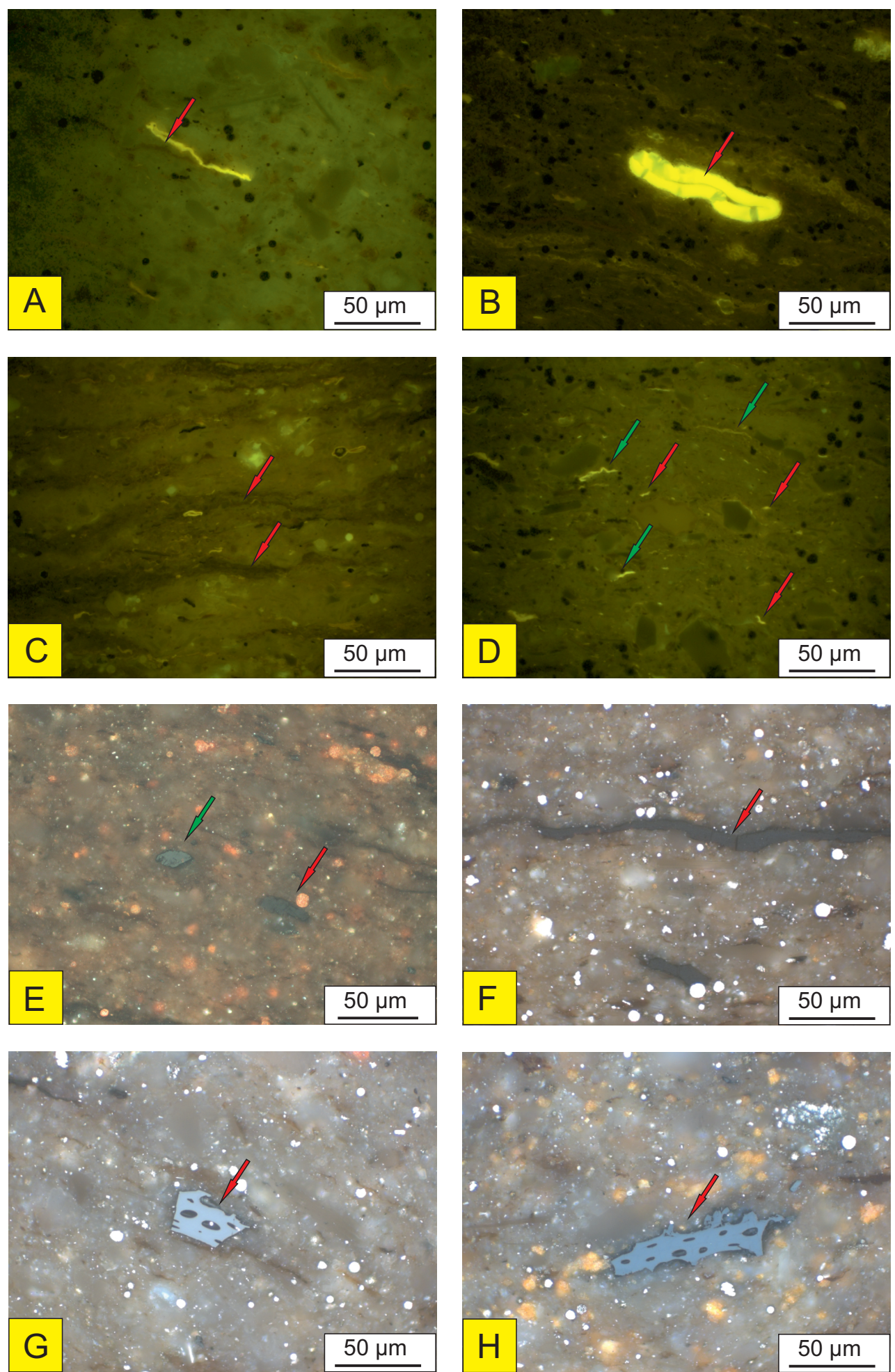

H. Komańcza-2, sample 2. Fragment of semifusinite with preserved cell structure (arrow). Reflected light

H. Komańcza 2, próbka 2. Fragment semifuzynitu z zachowaną strukturą tkankową (strzałka). Światło odbite

of organic matter, exhibiting yellow fluorescence - similar to alginite (plate I, D). It is probably a product of mechanical degradation of algae. It is a common, although due to its small size its content does not exceed about $2.5 \mathrm{vol} \%$.
The vitrinite group is represented mainly by collotelinite and witrodetrinite. Collotelinite occurs in the form of elongated fragments of various sizes. The length of collotelinite fragments varies from very small up to several hundred $\mu \mathrm{m}$, and its thick- 
ness usually does not exceed several tens of $\mu \mathrm{m}$ (plate I, E, F). It exhibits more or less intense brown fluorescence.

Fragments of collotelinite show significant diversity in terms of the reflectance (plate I, E). Usually the largest population of vitrinite particles have darker color with reflectance below $0.2 \%$ (so-called "saprovitrinite" or "dark vitrinite", ICCP, 1998). The next group consists of fragments with reflectance being in the range of $0.2-0.5 \%$. For these fragments reflectance was measured to determine the degree of maturity of organic matter. Both vitrinite groups are observed in the form of more or less elongated fragments. The next group are fragments of the so-called reworked vitrinite, characterized by high reflectance in the range of $0.6-1 \%$. Most times they have oval shape, and their size is usually small.

Collotelinite is usually accompanied by finer parts of vitrodetrinite, showing similar properties, but characterized by a particle size smaller than $10 \mu \mathrm{m}$. The content of all vitrinite fragments in the examined rocks is relatively low and reaches up to $1.6 \mathrm{vol} \%$.

Macerals of the inertinite group (fusinite, semifusinite, inertodetrinite) are usually limited to single occurrences within the entire investigated sample area. Fusinite is characterized by the highest reflectance (plate I, G). It occurs in the form of either multicellular structures or their fragments. Characteristic, sharp-edged fragments usually do not exceed several tens of micrometers, although sometimes larger accumulations in the form of laminae with a length of several hundred micrometers may also occur. Semifusinite in terms of the form of occurrence is very similar to that of fusinite, but it is characterized by lower reflectance (plate I, H). Inertodetrinite form fine $(<10 \mu \mathrm{m})$, sharp-edged fragments with high reflectance. None of the listed inertinite macerals exhibit fluorescence.

In 41 out of 43 samples, the collotelinite fragments were numerous enough that it was possible to measure their reflectance (Tab. 2). In other cases, either there was not enough representation of the vitrinite fragments, or its surface prevented proper measurements. For samples in which reflectance of vitrinite fragments was measured, this parameter ranges from $0.25 \%$ to $0.56 \%$. Rock-Eval pyrolysis results (Tab. 3) provided additional information, of which the most important (from the point of view of this study) were values of TOC, $\mathrm{T}_{\max }$ and hydrogen (HI) and oxygen (OI) indices. And so the $\mathrm{T}_{\max }$ parameter indicates temperatures in the range of $417-449^{\circ} \mathrm{C}$, the hydrogen index changes in wide range from 95 to 502, while the oxygen index does not exceed 133 .
Table 2. Results of vitrinite reflectance measurements

Tabela 2. Wyniki pomiarów refleksyjności witrynitu

\begin{tabular}{|c|c|c|c|c|}
\hline \multirow[b]{2}{*}{ Outcrop } & \multirow{2}{*}{$\begin{array}{l}\text { Sample } \\
\text { ID }\end{array}$} & \multicolumn{3}{|c|}{ Vitrinite reflectance } \\
\hline & & $\begin{array}{c}\mathbf{R}_{\mathbf{o}} \\
{[\%]}\end{array}$ & $\begin{array}{l}\text { standard } \\
\text { deviation }\end{array}$ & $\begin{array}{c}\text { number of } \\
\text { measurements }\end{array}$ \\
\hline \multirow{4}{*}{$\begin{array}{l}\text { Świątkowa } \\
\text { Wielka }\end{array}$} & 1 & 0.56 & 0.09 & 17 \\
\hline & 2 & 0.56 & 0.06 & 55 \\
\hline & 3 & 0.53 & 0.07 & 56 \\
\hline & 4 & 0.51 & 0.09 & 59 \\
\hline \multirow{5}{*}{ Mszana } & 1 & 0.28 & 0.05 & 10 \\
\hline & 2 & 0.34 & 0.06 & 51 \\
\hline & 3 & 0.33 & 0.04 & 13 \\
\hline & 4 & 0.36 & 0.06 & 50 \\
\hline & 5 & 0.36 & 0.07 & 31 \\
\hline \multirow{6}{*}{ Zyndranowa } & 1 & 0.32 & 0.08 & 48 \\
\hline & 2 & 0.28 & 0.06 & 17 \\
\hline & 3 & 0.29 & 0.08 & 24 \\
\hline & 4 & 0.28 & 0.07 & 13 \\
\hline & 5 & 0.28 & 0.05 & 25 \\
\hline & 6 & 0.30 & 0.06 & 51 \\
\hline \multirow{6}{*}{ Wisłok Wielki } & 1 & 0.29 & 0.06 & 20 \\
\hline & 2 & $\mathrm{x}$ & $\mathrm{x}$ & $\mathrm{x}$ \\
\hline & 3 & 0.32 & 0.09 & 23 \\
\hline & 4 & 0.31 & 0.08 & 37 \\
\hline & 5 & 0.25 & 0.09 & 12 \\
\hline & 6 & 0.31 & 0.10 & 17 \\
\hline \multirow{5}{*}{ Izwór stream } & 1 & 0.25 & 0.06 & 46 \\
\hline & 2 & 0.27 & 0.06 & 16 \\
\hline & 3 & 0.25 & 0.03 & 51 \\
\hline & 4 & 0.28 & 0.04 & 52 \\
\hline & 5 & 0.26 & 0.06 & 24 \\
\hline \multirow{6}{*}{ Czystogarb } & 1 & 0.28 & 0.07 & 21 \\
\hline & 2 & 0.30 & 0.06 & 15 \\
\hline & 3 & 0.28 & 0.05 & 42 \\
\hline & 4 & $\mathrm{x}$ & $\mathrm{x}$ & $\mathrm{x}$ \\
\hline & 5 & 0.27 & 0.04 & 51 \\
\hline & 6 & 0.26 & 0.05 & 30 \\
\hline \multirow{5}{*}{ Komańcza-1 } & 1 & 0.27 & 0.04 & 51 \\
\hline & 2 & 0.33 & 0.05 & 52 \\
\hline & 3 & 0.27 & 0.04 & 52 \\
\hline & 4 & 0.28 & 0.05 & 48 \\
\hline & 5 & 0.28 & 0.05 & 38 \\
\hline \multirow{6}{*}{ Komańcza-2 } & 1 & 0.28 & 0.06 & 13 \\
\hline & 2 & 0.41 & 0.04 & 53 \\
\hline & 3 & 0.43 & 0.06 & 21 \\
\hline & 4 & 0.39 & 0.07 & 19 \\
\hline & 5 & 0.29 & 0.06 & 21 \\
\hline & 6 & 0.33 & 0,05 & 52 \\
\hline
\end{tabular}


Table 3. Results of Rock-Eval pyrolysis

Tabela 3. Wyniki pirolizy Rock-Eval

\begin{tabular}{|c|c|c|c|c|c|c|c|c|c|c|c|c|}
\hline \multirow{2}{*}{ Outcrop } & \multirow{2}{*}{$\begin{array}{c}\text { Sample } \\
\text { ID }\end{array}$} & $\mathbf{T}_{\max }$ & $S_{1}$ & $\mathbf{S}_{2}$ & $\mathbf{S}_{3}$ & \multirow{2}{*}{ PI } & PC & RC & TOC & \multirow{2}{*}{ HI } & \multirow{2}{*}{ OI } & MINC \\
\hline & & {$\left[{ }^{\circ} \mathbf{C}\right]$} & {$[\mathrm{mg} / \mathrm{g}]$} & {$[\mathrm{mg} / \mathrm{g}]$} & {$[\mathrm{mg} / \mathrm{g}]$} & & {$[\%]$} & {$[\%]$} & {$[\%]$} & & & {$[\%]$} \\
\hline \multirow{4}{*}{$\begin{array}{l}\text { Świątkowa } \\
\text { Wielka }\end{array}$} & 1 & 448 & 0.12 & 0.82 & 0.20 & 0.13 & 0.09 & 0.73 & 0.82 & 100 & 24 & 1.25 \\
\hline & 2 & 448 & 0.86 & 8.08 & 0.17 & 0.10 & 0.76 & 3.90 & 4.66 & 173 & 4 & 0.85 \\
\hline & 3 & 449 & 1.03 & 9.60 & 0.27 & 0.10 & 0.91 & 3.95 & 4.86 & 198 & 6 & 0.69 \\
\hline & 4 & 448 & 0.33 & 2.42 & 0.70 & 0.12 & 0.28 & 1.77 & 2.05 & 118 & 34 & 3.22 \\
\hline \multirow{5}{*}{ Mszana } & 1 & 429 & 0.57 & 9.14 & 0.58 & 0.06 & 0.84 & 0.98 & 1.82 & 502 & 32 & 4.80 \\
\hline & 2 & 424 & 0.27 & 5.05 & 0.59 & 0.05 & 0.48 & 1.08 & 1.56 & 324 & 38 & 4.15 \\
\hline & 3 & 430 & 0.05 & 0.66 & 0.50 & 0.07 & 0.09 & 0.38 & 0.47 & 140 & 106 & 7.85 \\
\hline & 4 & 431 & 0.12 & 1.90 & 0.65 & 0.06 & 0.20 & 0.89 & 1.09 & 174 & 60 & 4.46 \\
\hline & 5 & 429 & 0.11 & 2.23 & 0.39 & 0.05 & 0.22 & 0.87 & 1.09 & 205 & 36 & 5.92 \\
\hline \multirow{6}{*}{ Zyndranowa } & 1 & 432 & 0.13 & 7.81 & 3.39 & 0.02 & 0.83 & 2.94 & 3.77 & 207 & 90 & 0.19 \\
\hline & 2 & 431 & 0.50 & 10.62 & 1.75 & 0.05 & 1.02 & 2.44 & 3.46 & 307 & 51 & 0.11 \\
\hline & 3 & 436 & 0.05 & 2.66 & 2.15 & 0.02 & 0.32 & 1.73 & 2.05 & 130 & 105 & 0.38 \\
\hline & 4 & 430 & 0.60 & 12.98 & 1.83 & 0.04 & 1.23 & 2.82 & 4.05 & 320 & 45 & 0.12 \\
\hline & 5 & 432 & 0.60 & 11.21 & 1.67 & 0.05 & 1.07 & 2.46 & 3.53 & 318 & 47 & 0.10 \\
\hline & 6 & 430 & 0.68 & 21.32 & 3.07 & 0.03 & 1.99 & 4.05 & 6.04 & 353 & 51 & 2.60 \\
\hline \multirow{6}{*}{$\begin{array}{l}\text { Wisłok } \\
\text { Wielki }\end{array}$} & 1 & 420 & 0.15 & 11.96 & 0.40 & 0.01 & 1.07 & 2.73 & 3.80 & 315 & 11 & 0.13 \\
\hline & 2 & 428 & 0.14 & 0.74 & 0.88 & 0.16 & 0.14 & 0.52 & 0.66 & 112 & 133 & 2.35 \\
\hline & 3 & 417 & 0.29 & 9.98 & 0.73 & 0.03 & 0.94 & 3.54 & 4.48 & 223 & 16 & 0.20 \\
\hline & 4 & 430 & 0.07 & 0.75 & 0.37 & 0.08 & 0.10 & 0.41 & 0.51 & 147 & 73 & 3.47 \\
\hline & 5 & 424 & 0.16 & 6.98 & 0.18 & 0.02 & 0.63 & 1.85 & 2.48 & 281 & 7 & 0.09 \\
\hline & 6 & 420 & 0.20 & 15.78 & 0.33 & 0.01 & 1.38 & 2.32 & 3.70 & 426 & 9 & 0.10 \\
\hline \multirow{5}{*}{ Izwór stream } & 1 & 423 & 0.22 & 14.36 & 0.21 & 0.02 & 1.25 & 1.65 & 2.90 & 495 & 7 & 0.08 \\
\hline & 2 & 424 & 0.35 & 18.82 & 0.21 & 0.02 & 1.64 & 2.20 & 3.84 & 490 & 5 & 0.10 \\
\hline & 3 & 431 & 0.02 & 0.82 & 0.81 & 0.02 & 0.11 & 0.62 & 0.73 & 112 & 111 & 2.67 \\
\hline & 4 & 436 & 0.01 & 0.63 & 0.70 & 0.02 & 0.09 & 0.57 & 0.66 & 95 & 106 & 3.10 \\
\hline & 5 & 422 & 0.21 & 16.49 & 0.50 & 0.01 & 1.45 & 2.70 & 4.15 & 397 & 12 & 0.16 \\
\hline \multirow{6}{*}{ Czystogarb } & 1 & 432 & 0.25 & 12.51 & 1.45 & 0.02 & 1.15 & 2.16 & 3.31 & 378 & 44 & 0.16 \\
\hline & 2 & 434 & 0.24 & 7.04 & 2.01 & 0.03 & 0.71 & 2.16 & 2.87 & 245 & 70 & 0.13 \\
\hline & 3 & 431 & 0.30 & 7.09 & 1.24 & 0.04 & 0.68 & 1.67 & 2.35 & 302 & 53 & 0.25 \\
\hline & 4 & 433 & 0.07 & 1.23 & 0.31 & 0.05 & 0.13 & 0.35 & 0.48 & 256 & 65 & 0.04 \\
\hline & 5 & 429 & 0.26 & 11.40 & 1.44 & 0.02 & 1.05 & 2.11 & 3.16 & 361 & 46 & 0.11 \\
\hline & 6 & 433 & 0.10 & 4.59 & 1.71 & 0.02 & 0.48 & 1.84 & 2.32 & 198 & 74 & 0.15 \\
\hline \multirow{5}{*}{ Komańcza-1 } & 1 & 426 & 0.52 & 16.12 & 0.26 & 0.03 & 1.41 & 2.66 & 4.07 & 396 & 6 & 0.13 \\
\hline & 2 & 425 & 0.87 & 20.61 & 0.70 & 0.04 & 1.84 & 3.21 & 5.05 & 408 & 14 & 0.17 \\
\hline & 3 & 425 & 1.63 & 17.52 & 0.10 & 0.09 & 1.62 & 3.02 & 4.64 & 378 & 2 & 0.18 \\
\hline & 4 & 432 & 0.35 & 4.61 & 1.77 & 0.07 & 0.50 & 1.91 & 2.41 & 191 & 73 & 0.15 \\
\hline & 5 & 427 & 0.24 & 6.71 & 1.44 & 0.03 & 0.66 & 1.75 & 2.41 & 278 & 60 & 0.16 \\
\hline \multirow{6}{*}{ Komańcza-2 } & 1 & 437 & 0.66 & 10.86 & 0.26 & 0.06 & 0.98 & 1.83 & 2.81 & 386 & 9 & 0.40 \\
\hline & 2 & 435 & 0.21 & 1.96 & 0.45 & 0.10 & 0.21 & 0.85 & 1.06 & 185 & 42 & 2.41 \\
\hline & 3 & 435 & 0.07 & 0.61 & 0.41 & 0.11 & 0.08 & 0.52 & 0.60 & 102 & 68 & 3.21 \\
\hline & 4 & 433 & 0.28 & 1.70 & 0.31 & 0.14 & 0.19 & 0.70 & 0.89 & 191 & 35 & 3.09 \\
\hline & 5 & 434 & 0.31 & 11.91 & 0.18 & 0.03 & 1.04 & 2.09 & 3.13 & 381 & 6 & 0.16 \\
\hline & 6 & 439 & 0.45 & 2.74 & 0.70 & 0.14 & 0.30 & 1.18 & 1.48 & 185 & 47 & 1.17 \\
\hline
\end{tabular}




\section{Discussion}

\section{Relations between maceral composition, lithological types and basic geochemical parameters}

The maceral composition in most of the samples is represented by the association of macerals:

collotelinite + vitrodetrinite + alginite + bituminite +

+ liptodetrinite + fusinite/semifusinite/inertodetrinite

Taking this into consideration, the interpretation draws attention not so much to the qualitative composition, which is rather similar, as to the varying proportions between the macerals, as here more distinct differences occur. The average content of individual macerals in relation to lithology is shown in Fig. 3.

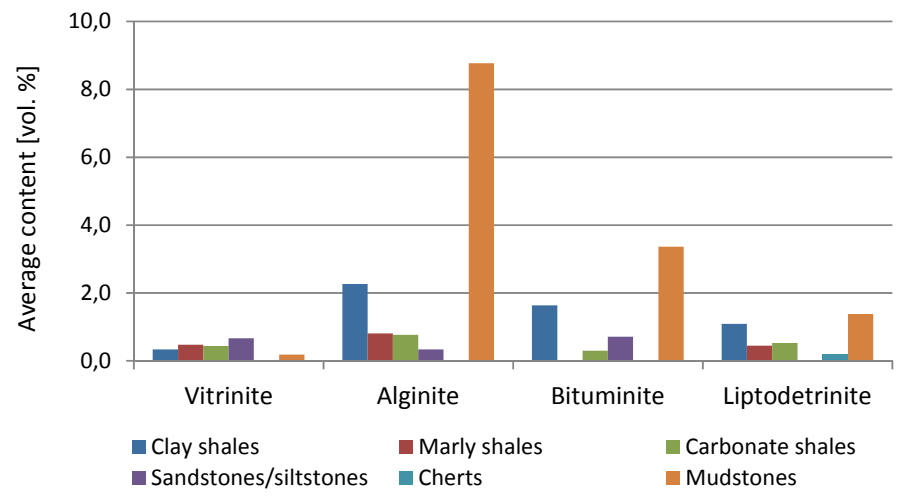

Fig. 3. Average content of macerals in relation to lithology

Fig. 3. Średni udział macerałów w odniesieniu do wykształcenia litologicznego

Mudstones have the highest average content of alginite, which reaches $9 \mathrm{vol} \%$. Average contents of bituminite and liptodetrinite (3.3 and 1.4 vol.\% respectively) are also the highest of all analyzed lithologies. Clay shales have a wide range of alginite content, from 0.2 to $13.2 \mathrm{vol} \%$, with an average value of $2.3 \mathrm{vol} \%$. Average contents of bituminite and liptodetrinite are 1.6 and $1.1 \mathrm{vol} \%$ respectively. These values are lower than those observed for mudstones, but still higher than in case of all other lithologies. In case of marly shales, sandstones/siltstones, micrite limestones and chert the content of individual liptinite macerals is always lower than $1 \mathrm{vol} \%$. The average content of vitrinite macerals in all analyzed lithologies does not exceed $1 \mathrm{vol} \%$. Their highest content is observed in sandstone sample (1.6 vol\%). Inertinite group, irrespective to lithology, is represented only by single fragments of fusinite, semifusinite and inertodetrinite.

In lithological interpretations it is necessary to refer also to the parameters obtained from Rock-Eval pyrolysis (TOC, HI, OI). These are summarized in Table 4.

The presented data shows that clay shales have the highest average TOC of $3.4 \%$, which is slightly higher compared to
Table 4. Values of TOC, HI, OI in relation to lithology

Tabela 4. Wartości parametrów TOC, HI, OI w odniesieniu do litologii

\begin{tabular}{|c|c|c|c|c|}
\hline Lithology & Value & $\begin{array}{c}\text { TOC } \\
{[\%]}\end{array}$ & HI & OI \\
\hline \multirow{3}{*}{ Clay shales } & Min. & 0.82 & 100 & 2 \\
\hline & Max. & 5.05 & 495 & 105 \\
\hline & Average & 3.43 & 303 & 33 \\
\hline \multirow{3}{*}{ Marly shales } & Min. & 0.73 & 112 & 34 \\
\hline & Max. & 6.04 & 353 & 111 \\
\hline & Average & 2.58 & 192 & 61 \\
\hline \multirow{3}{*}{$\begin{array}{l}\text { Carbonate shales } \\
\text { and micritic } \\
\text { limestones }\end{array}$} & Min. & 0.47 & 102 & 32 \\
\hline & Max. & 1.82 & 502 & 106 \\
\hline & Average & 0.94 & 206 & 57 \\
\hline \multirow{3}{*}{ Mudstones } & Min. & 2.41 & 278 & 6 \\
\hline & Max. & 3.16 & 381 & 60 \\
\hline & Average & 2.90 & 340 & 37 \\
\hline \multirow{3}{*}{$\begin{array}{l}\text { Sandstones, } \\
\text { siltstones }\end{array}$} & Min. & 0.66 & 95 & 38 \\
\hline & Max. & 1.56 & 324 & 133 \\
\hline & Average & 0.96 & 177 & 92 \\
\hline Chert & $\mathrm{x}$ & 0.48 & 256 & 65 \\
\hline
\end{tabular}

mudstones (2.9\%) and marly shales (2.6\%). For other lithologies, the average value of this parameter does not exceed $1 \%$. The average value of the hydrogen index (HI) only in the case of clay shales and mudstones exceeds 300 (303 and 340, respectively). For other lithologies, the average value of this parameter is in the range 177-256 (the lowest for sandstones/ siltstones). Oxygen index (OI) values behave inversely to the hydrogen index and for mudstones and clay shales, the average value of this parameter is the lowest ( 37 and 33 respectively), while for sandstones/siltstones it is the highest and reaches 92 . For other lithologies, the average value of this parameter in the range of 50-65.

\section{Organic matter maturity and kerogen type}

In case of assessing the thermal maturity of organic matter, two parameters were used - vitrinite reflectance $R_{o}$ and the $T_{\max }$ parameter. Both of these parameters clearly indicate some variation in the degree of transformation of organic matter within the studied area.

In the western part, within the tectonic window of Światkowa, the Menilite Beds are in the phase of oil window, as evidenced by $\mathrm{T}_{\max }$ of $448^{\circ} \mathrm{C}$ and $\mathrm{R}_{0}$ reaching $0.54 \%$. In none of the other locations, such a high degree of organic matter transformation is observed. The average $\mathrm{T}_{\max }$ in the remaining area is in the range of $427-436^{\circ} \mathrm{C}$ which suggest the immature phase or exceptionally the border between the immature phase and the beginning of the oil window. The average vitrinite 


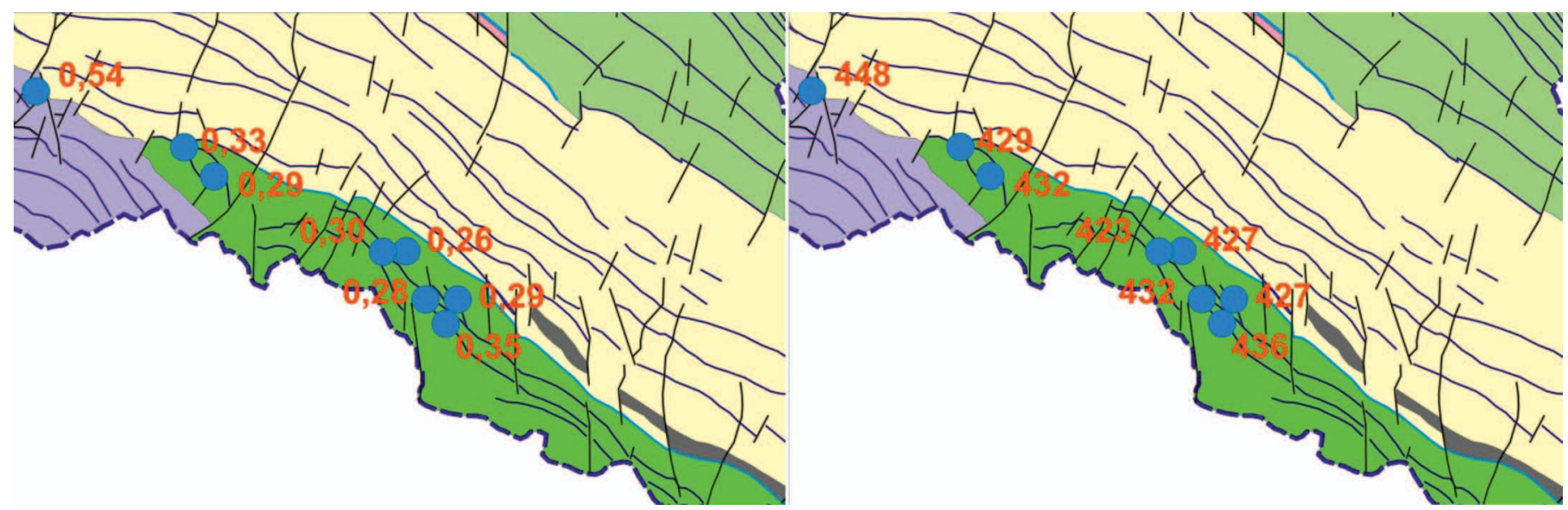

Fig. 4. Average vitrinite reflectance (left) and $\mathrm{T}_{\max }$ (right) for the investigated outcrops

Rys. 4. Średnia refleksyjność witrynitu (po lewej) oraz średni $T_{\max }$ (po prawej) dla poszczególnych odsłonięć na przebadanym obszarze

reflectance is in range of 0.26 to 0.33 , which indicates the immaturity of the organic matter. Both $\mathrm{T}_{\max }$ and $\mathrm{R}_{\mathrm{o}}$ differs very little within the analyzed area which make it impossible to draw any geographic trends of these changes (Fig. 4).

Analyzed samples contain type II, II/III or III of kerogen (Fig. 5, 6). Type II dominates in clay shales and mudstones, while type III is most often observed in marly and carbonate shales and also in sandstones/siltstones.

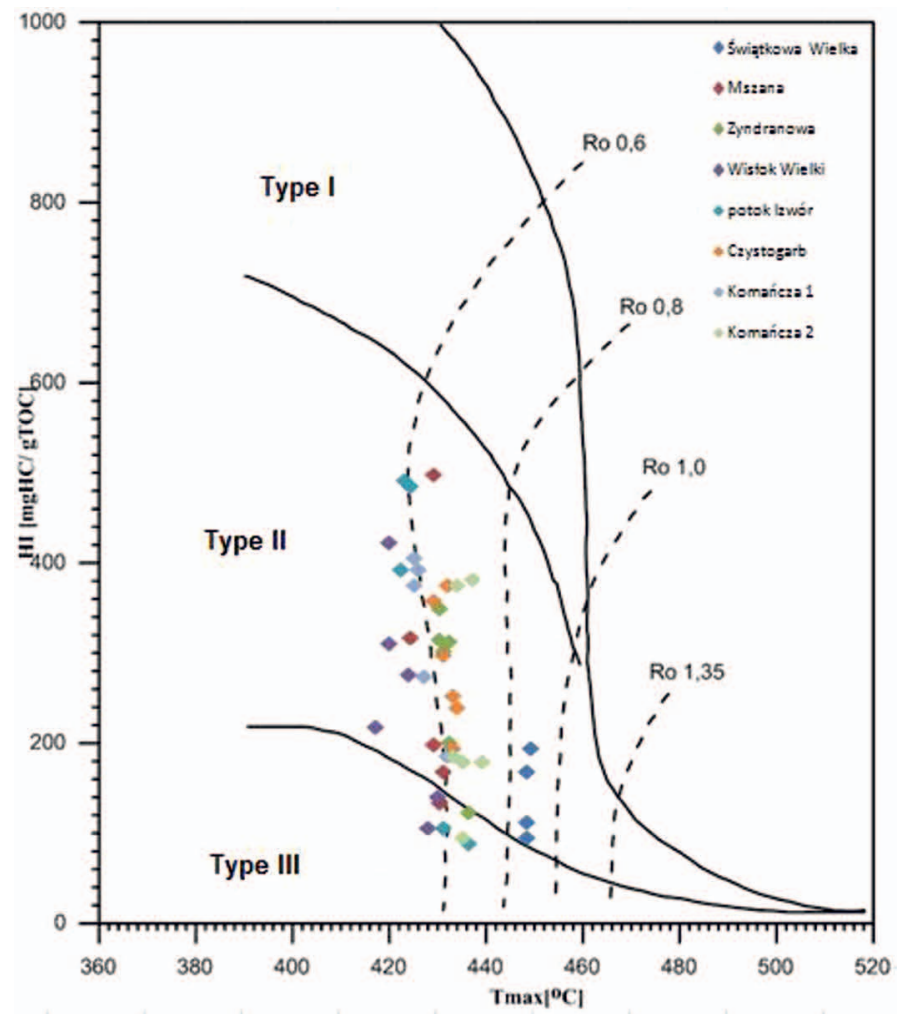

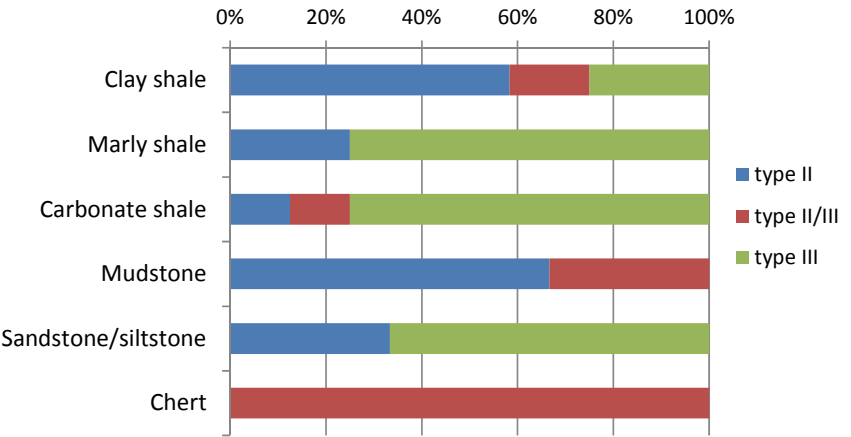

Fig. 5. Kerogen types in relation to lithology

Rys. 5. Typ kerogenu w wydzieleniach litologicznych

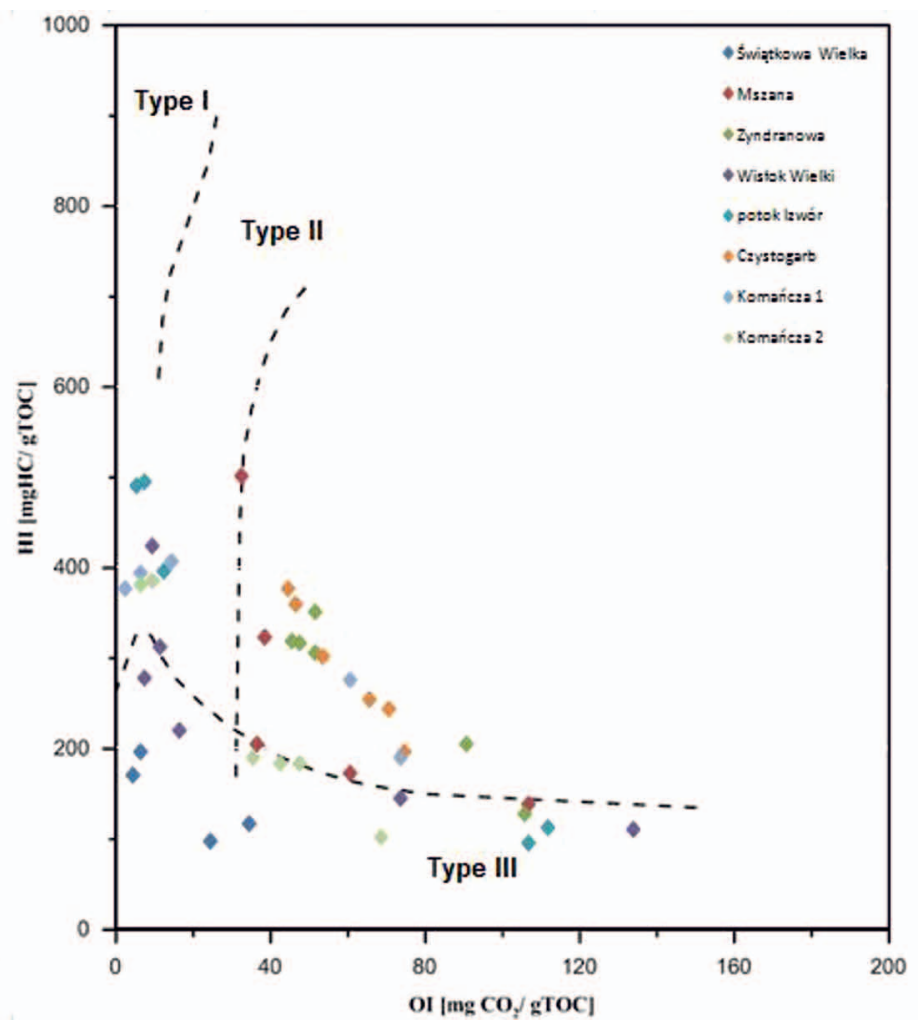

Fig. 6. Types of kerogen $-\mathrm{T}_{\max }-\mathrm{HI}$ (left) and OI-HI (right) diagrams

Rys. 6. Typy kerogenu - diagram $\mathrm{T}_{\max }-\mathrm{HI}$ (po lewej) oraz OI-HI (po prawej) 


\section{Summary}

The composition of the organic matter of the Menilite Beds within the Dukla Unit is dominated by macerals belonging to the liptinite group - alginite, bituminite and liptodetrinite. Macerals of the vitrinite (collotelinite, vitrodetrinite) and inertinite (fusinite, semifusinite, inertodetrinite) are also present, but their content is rather low. The richest in organic matter are clay shales and mudstones, while other lithologies are characterized by lower content of organic fragments. Analyzed organic matter is most often in the immature phase, a greater degree of thermal transformation, corresponding to so-called oil window is observed only in the Świątkowa tectonic window in the western part of the studied area. Composition of organic matter corresponds to the types II and III (and their mixture) of the kerogen.

ACKNOWLEDGES: The author would like to thank Leszek Jankowski for his advice on outcrop selection.

This paper was written on the basis of the statutory work entitled: Characteristics of the dispersed organic matter in the Menilite Beds from the Dukla Unit - the work of the Oil and Gas Institute - National Research Institute was commissioned by the Ministry of Science and Higher Education; order number: 52/SG/18, archival number: SG-4101-52/18.

\section{Literature}

Curtis J.B., Kotarba M.J., Lewan M.D., Więcław D., 2004. Oil/ source rock correlations in the Polish Flysch Carpathians and mesozoic basement and organic facies of the oligocene menilite shales: insights from hydrobus pyrolysis experiments Organic Geochemistry, 35: 1573-1596. DOI:10.1016/j. orggeochem.2004.06.018.

International Committee for Coal and Organic Petrology (ICCP), 1998. The new vitrinite classification (ICCP System 1994). Fuel, 77(5): 349-358.

Jankowski L., 2008. Kompleksy chaotyczne Karpat Polskich. Materiały konferencyjne „Przewodnik sesji terenowej”, Kraków - Polańczyk 2008, 26-88.

Jankowski L., Probulski J., 2011. Rozwój tektoniczno-basenowy Karpat zewnętrznych na przykładzie budowy geologicznej złóż Grabownica, Strachocina i Łodyna oraz ich otoczenia. Geologia, 37: 555-583.

Koltun Y.V., 1992. Organic matter in oligocene Menilite formation rocks of the Ukrainian Carpathians: palaeoenvironment and geochemical evolution. Organic Geochemistry, 18: 423-430. DOI: 10.1016/0146-6380(92)90105-7.

Koltun Y., Espitalie J., Kotarba M., Roure F., Ellouz N., Kosakowski P., 1998. Petroleum generation in the Ukrainian external Carpathians and the adjacent foreland. Journal of Petroleum Geology, 21(3): 265-288. DOI: 10.1111/j.1747-5457.1998.tb00782.x.

Kosakowski P., Koltun Y., Machowski G., Papiernik B., 2018. The geochemical characteristics of the Oligocene-lower Miocene menilite formation in the Polish and Ukrainian Outer Carpathians: A review. Journal of Petroleum Geology, 41(3): 319-335. DOI: 10.1111/jpg.12705.
Kosakowski P., Więcław D., Kotarba M.J., 2009. Charakterystyka macierzystości wybranych utworów fliszowych w przygranicznej strefie polskich Karpat Zewnętrznych. Geologia, 35(4/1): 155-190.

Köster J., Kotarba M., Lafargue E., Kosakowski P., 1998a. Source rock habitat and hydrocarbon potential of oligocene menilite formation (Flysch Carpathians, Southeast Poland): an organic geochemical and isotope approach. Organic Geochemistry, 29: 543-558. DOI: 10.1016/S0146-6380(98)00059-X.

Köster J., Rospondek M., Kotarba M., Zubrzycki A., de Leeuw J.W., Sinninghe Damsté J.S., 1996. Biological markers and stable carbon isotope ratios in palaeoenvironmental reconstructions: examples from the Menilite Formation (Oligocene) of the Flysch Carpathians. Sediment'96. 11. Sedimentologen-Treffen, 83.

Köster J., Rospondek M., Schouten S., Kotarba M., Zubrzycki A., Sinninghe Damste J.S., 1998b. Biomarker geochemistry of a foreland basin: the Oligocene Menilite Formation in the Flysch Carpathians of Southeast Poland. Organic Geochemistry, 29: 649-669. DOI: 10.1016/S0146-6380(98)00182-X.

Köster J., Rospondek M.J., Zubrzycki A., Kotarba M., de Leeuw J.W., Sinninghe Damsté J.S., 1995. A molecular organic geochemical study of black shales associated with diatomites from the Oligocene Menilite Shales (Flysch Carpathians, SE Poland). [in:] Organic Geochemistry: Developments and Applications to Energy, Climate, Environment and Human History. Eds. J.O. Grimalt, C. Dorronsoro. A.I.G.O.A: 87-89.

Kotarba M.J., Curtis J.B., Lewan M.D., 2009. Comparison of natural gases accumulated in Oligocene strata with hydrous pyrolysis gases from Menilite Shales of the Polish Outer Carpathians. Organic Geochemistry, 40: 769-783. DOI: 10.1016/j. orggeochem.2009.04.007.

Kotarba M.J., Koltun Y.V., 2006. Origin and habitat of hydrocarbons of the Polish and Ukrainian parts of the Carpathian Province. [in:] Golonka J., Picha F. Eds. The Carpathians: Geology and Hydrocarbon Resources. American Association of Petroleum Geologists Memoir, 84: 395-443.

Kotarba M.J., Lewan M.D., 2002. Hydrocarbon potential of Oligocene Menilite formation of the Polish Flysch Carpathians by hydrous pyrolysis and stable isotopes. US-Poland Maria Sklodowski-Curie Joint Fund II-Project Report MEN/USGS-97-319.

Kotarba M.J., Lewan M.D., Wiecław D., Curtis J.B., 2005. Stable carbon isotope fractionation and organic sulphur content in hydrous pyrolysis $\left(24-108 \mathrm{~h}\right.$ and $\left.300-365^{\circ} \mathrm{C}\right)$ immiscible oils, bitumens and kerogens from the Oligocene Menilite Shales of the Polish Flysch Carpathians. Seville, Spain: $22^{\text {nd }}$ International Meeting on Organic Geochemistry: 338-339.

Kotarba M.J., Nagao K., 2008. Composition and origin of natural gases accumulated in the Polish and Ukrainian parts of the Carpathian region: gaseous hydrocarbons, noble gases, carbon dioxide and nitrogen. Chemical Geology, 255: 426-438. DOI: 10.1016/j. chemgeo.2008.07.011.

Kotarba M.J., Wiecław D., Koltun Y.V., Marynowski L., Kuśmierek J., Dudok I.V., 2007. Organic geochemical study and genetic correlation of natural gas, oil and Menilite source rocks in the area between San and Stryi rivers (Polish and Ukrainian Carpathians). Organic Geochemistry, 38(8): 1431-1456. DOI: 10.1016/j.orggeochem.2007.03.012

Kotarba M.J., Więcław D., Dziadzio P., Kowalski A., Bilkiewicz E., Kosakowski P., 2013. Organic geochemical study of source rocks and natural gas and their genetic correlation in the central part of the Polish Outer Carpathians. Marine and Petroleum Geology, 45: 106-120. DOI: 10.1016/j.marpetgeo.2013.04.018.

Kotarba M.J., Więcław D., Dziadzio P., Kowalski A., Kosakowski P., Bilikiewicz E., 2014. Organic geochemical study of source rocks 
and natural gas and their genetic correlation in the central part of the Polish Outer Carpathians and Paleozoic-Mesozoic basement. Marine and Petroleum Geology, 56: 97-122. DOI: 10.1016/j. marpetgeo.2014.03.014.

Kotulová J., 2004: Oligocene Menilite black shales - geochemical and maceral analysis. Florence: $32^{\text {nd }}$ International Geological Congress: 1-752.

Kruge M.A., Mastalerz M., Solecki A., Stankiewicz B.A., 1996. Organic geochemistry and petrology of oil source rocks, Carpathian Overthrust region, southeastern Poland - implications for petroleum generation. Organic Geochemistry, 24: 897-912. DOI: 10.1016/S0146-6380(96)00067-8.

Lewan M.D., Kotarba M.J., Curtis J.B., Więcław D., Kosakowski P., 2006. Oil-generation kinetics for organic facies with Type-II and-IIS kerogen in the Menilite Shales of the Polish Carpathians. Geochimica et Cosmochimica Acta, 70: 3351-3368. DOI: 10.1016/j.gca.2006.04.024.

Matyasik I., 1994. Badania geochemiczne warstw menilitowych, inoceramowych i spaskich jednostki skolskiej fliszu karpackiego. Nafta-Gaz, 40: 234-243.

Matyasik I., Kupisz L., 1996. Geological and geochemical conditions of the hydrocarbon generation in the Menilite Beds from the south part of the Strzyżów Depression. Janowice: The $2^{\text {nd }}$ Conference on the Geochemical and Petrophysical Investigations in Oil and Gas Exploration: 179-197.

Matyasik I., Steczko A., Mastalerz M., Brassell S.C., 2001. Petrographic and geochemical characterization of source rock variability in the Carpathian region of Poland: implication for oil generation. [in:] Abstracts of Eighteenth Annual Meeting of the Society for Organic Petrology, 18: 78-83.

Pawlewicz M., 2006. Total Petroleum Systems of the North Carpathian Province of Poland, Ukraine, Czech Republic and Austria. U.S. Geological Survey Bulletin 2204-D, 26.

Sachsenhofer R., Koltun Y., 2012. Black shales in Ukraine - A review. Marine and Petroleum Geology, 31: 125-136. DOI: 10.1016/j. marpetgeo.2011.08.016.

Semyrka G., 2009. Refleksyjność witrynitu i typy kerogenu w profilach wierceń wschodniej części Karpat polskich. Geologia, 35(2/1): 49-59.

Świdziński H., 1947. Słownik stratygraficzny północnych Karpat fliszowych. Biuletyn Instytutu Geologicznego, 124.

ten Haven H.L., Lafargue E., Kotarba M., 1993. Oil/oil and oil/source rock correlations in the Carpathian Foredeep and Overthrust, south-east Poland. Organic Geochemistry, 20: 935-959. DOI: 10.1016/0146-6380(93)90105-K.
Vetö I., Hetényi, M., 1991. Fate of Organic carbon and reduced sulphur in dysoxic-anoxic Oligocene facies of the Central Paratethys (Carpathian Mountains and Hungary). In: Modern and Ancient Continental Shelf Anoxia. Eds. R.V. Tyson, T.H. Pearson. London: Geological Society Special Publication, 58: 449-460.

Waliczek M., Machowski G., Świerczewska A., 2017. Bitumen in rocks from the Skrzydlna Thrust Sheet and the Mszana Tectonic Window (Outer Carpathians). Mineralogia - Special Papers, 46.

Wendorff M., Rospondek M.J., Kluska B., Marynowski L., 2017. Organic maturity and hydrocarbon potential of the Lower Oligocene Menilite facies in the Eastern Flysch Carpathians (Turcău and Vrancea Nappes), Romania. Applied Geochemistry, 78: 295-310. DOI: 10.1016/j.apgeochem.2017.01.009.

Więcław D., 2002. Geneza oligoceńskiej ropy naftowej polskich Karpat fliszowych - siarka organiczna w kerogenie warstw menilitowych a kinetyka procesu generowania węglowodorów. Kraków: $\mathrm{PhD}$ thesis, Akademia Górniczo-Hutnicza im. St. Staszica.

Więcław D., Kotarba M.J., Kuśmierek J., Kowalski A., Machowski G., 2008. Pionowa zmienność wskaźników macierzystości warstw menilitowych w wybranych profilach wschodniej części polskich Karpat Zewnętrznych. Prace Instytutu Nafty i Gazu, 150, 455-460.

Zielińska M., 2012. Petrologiczne stadium uwęglonego materiału organicznego we fliszu zewnętrznych Karpat Zachodnich. Kraków: $\mathrm{PhD}$ thesis, Akademia Górniczo-Hutnicza im. St. Staszica.

Ziemianin K., 2018. Characteristics of dispersed organic matter in the Menilite Beds from the Skole Unit. Nafta-Gaz, 9: 636-646. DOI: 10.18668/NG.2018.09.02.

Ziemianin, K., 2017. Petrographic-geochemical characterization of the dispersed organic matter in Menilite shales from the Silesian Unit in the Carpathian Mountains of SE Poland. Nafta-Gaz, 11: 835-842. DOI: 10.18668/NG.2017.11.02.

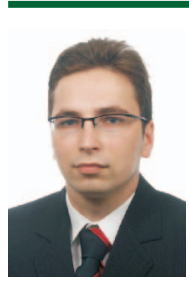

Konrad ZIEMIANIN M.Sc.

Assistant at the Geology and Geochemistry Department

Oil and Gas Institute - National Research Institute 25 A Lubicz St.

31-503 Krakow

E-mail:konrad.ziemianin@inig.pl 